\title{
Phytomelatonin: a plant hormone for management of stress
}

\begin{abstract}
Today different stresses arise due to climate change or due to natural and manmade activities. Water stress and heat stress (global warming) occurs due to manmade activities like deforestation and urbanization. The science behind plants stress tolerance to biotic and abiotic stresses is always remains interesting and important aspect for researchers to enhance the crop productivity in arid and semiarid regions all over the world. The article reviews the role of Phytomelatonin in plant stress tolerance. Phytomelatonin ( $N$-acetyl-5methoxytryptamine) is indoleamine related to tryptophan involve in many physiological activities of plant. Phytomelatonin may be endogenous or exogenously applied to improve the plants stress tolerance against different types of abiotic and biotic stresses, including drought, salinity, chilling, heat and fungal pathogens. It acts as a signaling molecule in plant responses to stress by inducing a number of defense genes through SA, GA, ABA signaling pathway. Serotonin $N$-acetyltransferase (SNAT) is the essential gene in phytomelatonin biosynthesis. If the plants modified genetically by incorporating gene for SNAT metabolism, the plants will synthesis Phytomelatonin which helps them to withstand in harsh environmental stresses. This review explains that phytomelatonins potential role as antioxidant and relives biotic or abiotic stresses thereby stabilize membrane, increases photosynthetic activity and growth of plants.
\end{abstract}

Keywords: phytomelatonin; abiotic and biotic stresses; signaling molecule
Volume 7 Issue 2 - 2018

\author{
Archana M Dhole, Harsha N Shelat \\ Department of Agricultural Microbiology, Anand Agricultural \\ University, India
}

\author{
Correspondence: Archana M Dhole, BA College of \\ Agriculture, Department of Agricultural Microbiology, Anand \\ Agricultural University, Anand (Gujarat), India, \\ Email archanadhole.2009@gmail.com
}

Received: November 10, 2017| Published: April 09, 2018
Abbreviations: SNAT, serotonin $N$-acetyltransferase; IAA, indole-3-acetic acid; RIA, radioimmunoassay; ELISA, enzymelinked immunosorbent assay; GS-MS, gas chromatography-mass spectrometry; HPLC, high-performance liquid chromatography; ECD, electrochemical detection; FD, fluorescence detection; TDC, tryptophan decarboxylase; T5H, tryptophan 5-hydroxylase; 2-ODDS, 2-oxoglutarate-dependent dioxygenases; OMT, o-methyltransferase; ROS, reactive oxygen species; $\mathrm{OH}^{+}$, including hydroxyl radical; $\mathrm{NO}_{3}{ }_{3}$ , peroxynitrite anion; ${ }^{1} \mathrm{O}_{2}{ }^{-}$, , singlet oxygen; $\mathrm{NO}^{*}$, nitric oxide; RNS reactive nitrogen species; $\mathrm{SOD}$, superoxide dismutase; CAT, catalase; APX, ascorbate peroxidase; GSH-PX, glutathione peroxidase; GSSG-R, glutathione reductase

\section{Introduction}

Different biotic and abiotic stresses are adversely affect the growth and productivity of crop plants all over the world. So the study of stress tolerance has great importance in scientific community. Phytomelatonin, once discovered in vertebrates as a hormone of the pineal gland, is now known to be synthesized in bacteria and numerous taxa of eukaryotes including various algae of different phylogenetic position and plants.

It is biochemically $N$-acetyl-5-methoxytryptamine; biogenic indoleamine, structurally related to tryptophan, tryptamine, serotonin and indole-3-acetic acid (IAA). It is hormone involved in physiological activities. Plants accumulate high levels of phytomelatonin when faced with harsh environments ${ }^{1-3}$ and exogenously applied phytomelatonin helps to improve tolerance to stresses. Phytomelatonin ubiquitously present in organisms whose metabolism based on oxygen, so speculated that its primary function to protect organisms against oxygen toxicity.
Phytomelatonin in plants has been detected by several methods including radioimmunoassay (RIA), enzyme-linked immunosorbent assay (ELISA), gas chromatography-mass spectrometry (GSMS), and high-performance liquid chromatography (HPLC) with electrochemical detection (HPLC-ECD), fluorescence detection (HPLC-FD), or HPLC-MS. ${ }^{4}$

\section{Phytomelatonin synthesis}

Phytomelatonin synthesized from tryptophan with the help of tryptophan decarboxylase, tryptophan hydroxylase, tryptamine 5-hydroxylase, serotonin $\mathrm{N}$-acetyltransferase, $\mathrm{N}$-acetylserotonin methyltransferase, caffeic acid O-methyltransferase enzymes in different subcellular locations (Figure 1).

a. Tryptophan decarboxylase (TDC) is first committed enzyme for phytomelatonin biosynthesis in plants, catalyzes tryptophan into tryptamine or 5-hydroxytryptophan to serotonin. It is soluble cytosolic protein exists as a homodimer with a monomer molecular weight of 54,000. It shows high substrate specificity to tryptophan and 5-hydroxytryptophan.

b. Second step in phytomelatonin biosynthesis is catalyzed by Tryptophan 5-hydroxylase (T5H) and is associated with the hydroxylation reaction, which is predominantly mediated by cytochrome P450-dependent monooxygenases (P450s) and 2-oxoglutarate-dependent dioxygenases (2-ODDs) in plants. It also catalyse Tryptophan to 5-Hydroxytryptophan and $\mathrm{N}$-Acetyl tryptamine to $\mathrm{N}$-Acetyl Serotonin reactions.

c. The penultimate step in phytomelatonin biosynthesis is catalyzed by serotonin $N$-acetyltransferase SNAT (also known as arylalkylamine $N$-acetyltransferase, AANAT). It catalyzes transfer of an acetyl group from acetylcoenzyme A to an array 
of molecules, such as aminoglycosides and arylalkylamines. The rice SNAT enzyme can accept various amine substrates, such as serotonin, tryptamine, and 5-methoxytryptamine, which are catalyzed to $\mathrm{N}$-acetylserotonin, $\mathrm{N}$-acetyltryptamine, and phytomelatonin.

d. 5-hydroxyindol O-methyltranseferase is last step enzyme; it catalyzes $\mathrm{N}$-acetylserotonin into phytomelatonin by O-methyltransferase (OMT) reaction.

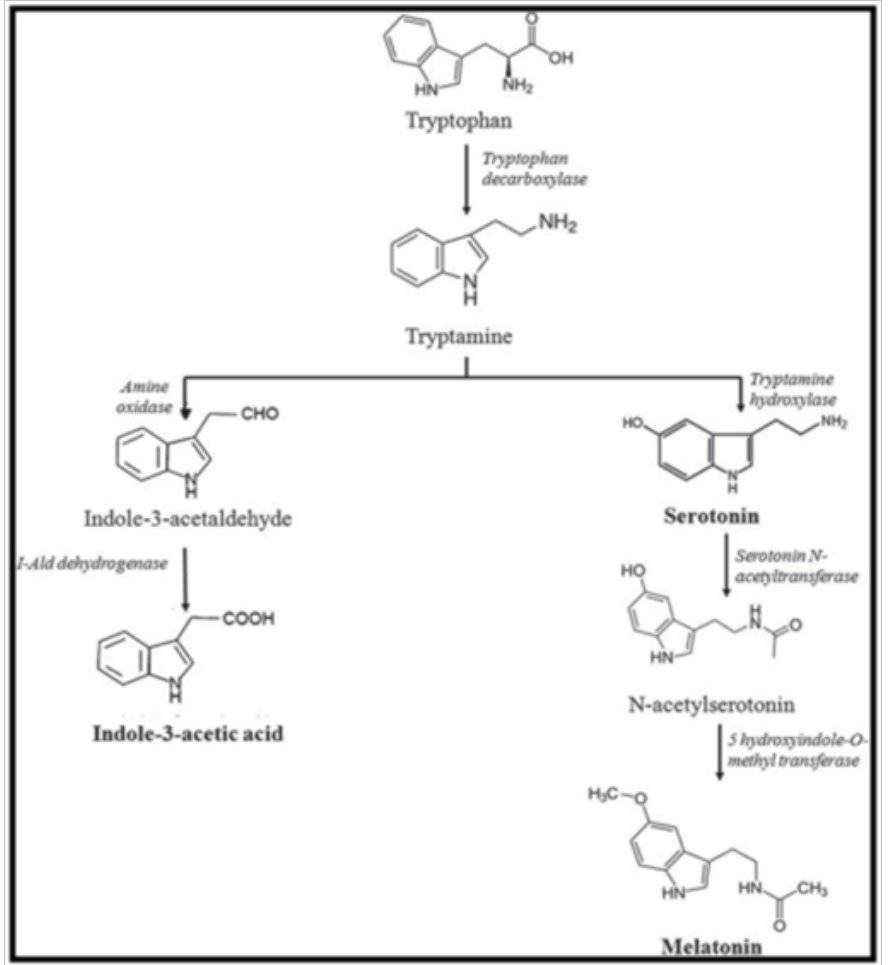

Figure I Biosynthesis of phytomelatonin in plants.

Source: Kaur et al. ${ }^{5}$

\section{Mode of action of phytomelatonin}

Phytomelatonin has greater antioxidative potential. Phytomelatonin molecule consists of an electron-rich indole moiety and 2 side chain groups: 5-methoxy group and 3-amide group (Figure 2). The high resonance stability, electroreactivity and low activation energy barriers make phytomelatonin a potent free radical scavenger. The side chains also have a significant contribution in the antioxidative properties of the molecule. Carbonyl moiety present in the functional group $(\mathrm{N}-\mathrm{C}=\mathrm{O})$ of $\mathrm{C} 3$ amide side chain has a key role in the quenching of more than one reactive oxygen species. After the interaction of phytomelatonin with ROS, the nitrogen present in the carbonyl group of the phytomelatonin molecule leads to the formation of a new 5 membered ring. ${ }^{5}$

It is an endogenous free radical scavenger and a broad-spectrum antioxidant. It detoxifies a variety of free radicals and reactive oxygen species (ROS) including hydroxyl radical $\left(\mathrm{OH}^{\circ}\right)$, peroxynitrite anion $\left(\mathrm{NO}_{3}^{-}\right)$, singlet oxygen $\left({ }^{1} \mathrm{O}_{2}^{-}\right)$and nitric oxide $\left(\mathrm{NO}^{\circ}\right)$. One of the most appealing properties of this molecule, which distinguishes it from most antioxidants, is the fact that its metabolites also have the ability to scavenge ROS and reactive nitrogen species (RNS).

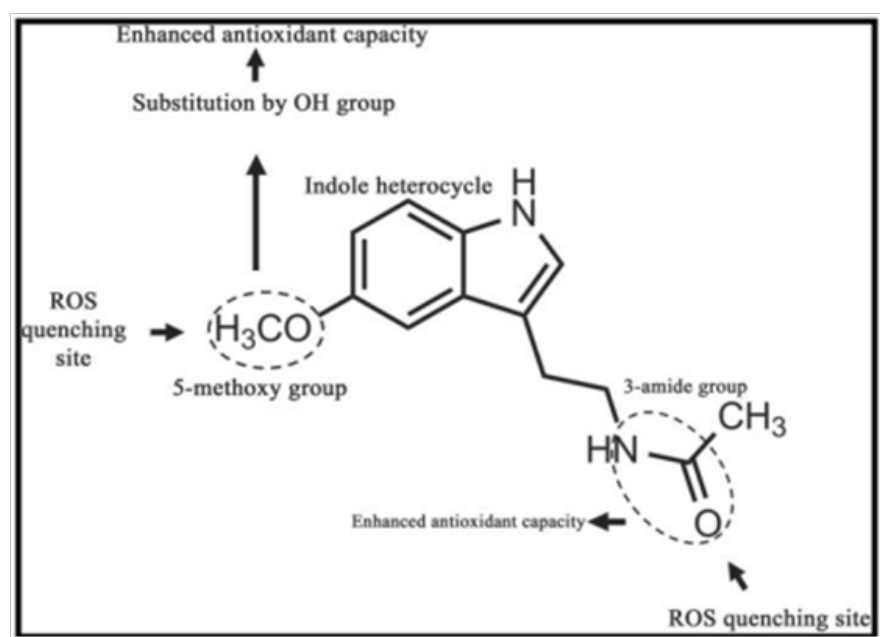

Figure 2 ROS quenching sites of Phytomelatonin.

Source: Kaur et al. ${ }^{5}$

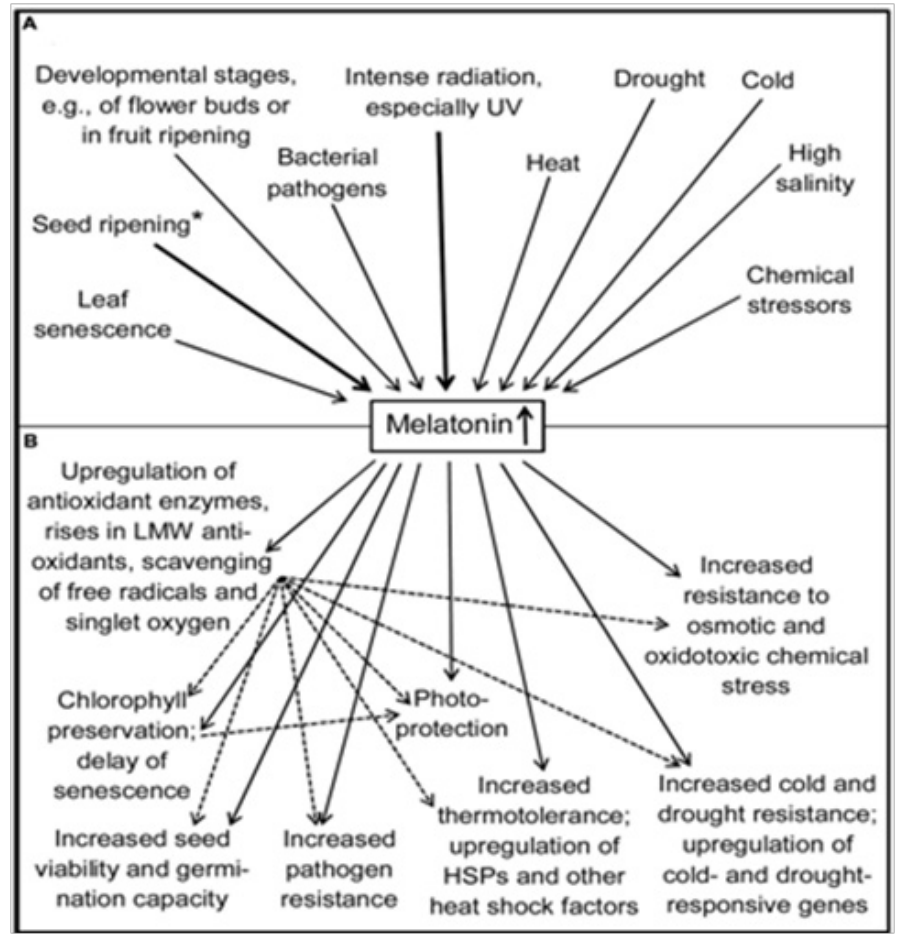

Figure 3 Increases in phytomelatonin (A) and its role in protection and stress tolerance (B).

Source: Hardeland ${ }^{2}$

Phytomelatonin generates free radical scavenging cascade providing continuous cell defense, even at low concentrations with highly effective protection from oxidative stress. Although phytomelatonin acts as a direct antioxidant it may also regulate activities of several antioxidant enzymes including superoxide dismutase (SOD), catalase (CAT), ascorbate peroxidase (APX), glutathione peroxidase (GSH-PX), glutathione reductase (GSSG-R).

\section{Role of phytomelatonin}

Phytomelatonin has proven to be ubiquitously synthesized in 
plant organs. Both abiotic and biotic stresses have been shown to induce phytomelatonin synthesis in plants. The compensatory rise in phytomelatonin under stressful conditions, in turn, helps to alleviate the negative consequences of stress, in particular oxidative stress, in the plants. Typically, stress of any type to plants compromises their growth which is often secondary to excessive generation of reactive oxygen species (ROS) including free radicals. Pleiotropic roles ranging from enhancing germination to delaying senescence of plants have been reported (Figure 3).

a. Delay senescence.

b. Promote growth.

c. Stimulate photosynthetic activity.

d. Protect plant against different types of abiotic and biotic stresses.

e. Participate in processes that affect plant development and reproduction.

f. Stabilize membrane.

g. Up and down regulate gene expression during different environmental conditions.

h. Enhances the phytoremediative capacity of plants.

\section{Conclusion}

Phytomelatonin ( $N$-acetyl-5-methoxytryptamine) biogenic indoleamine, a remarkably, heterogeneously-functioning beneficial molecule in plants. It synthesized from tryptophan with the help of tryptophan decarboxylase, tryptophan hydroxylase, tryptamine 5-hydroxylase, serotonin $\mathrm{N}$-acetyltransferase, $\mathrm{N}$-acetylserotonin methyltransferase, caffeic acid O-methyltransferase enzymes. It has greater antioxidative potential; have the ability to scavenge ROS and reactive nitrogen species (RNS). Its discovery in land plants two decades ago has led to a burgeoning investigative field that has already made substantial advances in uncovering the marked actions of this versatile indoleamine.

\section{Acknowledgements}

We are thankful to DST (Department of Science) Govt. of India, for providing a financial support through INSPIRE Fellowship for $\mathrm{Ph}$ D work.

\section{Conflict of interest}

The author declares that there is no conflict of interest.

\section{References}

1. Arnao MB, Ruiz JH. Functions of melatonin in plants: a review. J Pineal Res. 2015;59(2):133-150.

2. Rüdiger Hardeland. Melatonin in Plants-Diversity of Levels and Multiplicity of Functions. Front Plant Sci. 2016;7:198.

3. Zhang $\mathrm{H}$, Zhang $\mathrm{N}$, Yang $\mathrm{RC}$, et al. Melatonin promotes seed germination under high salinity by regulating antioxidant systems, ABA and GA4 interaction in cucumber (Cucumis sativus L.). J Pineal Res. 2014;57(3):269-279.

4. Nawaz MA, Huang Y, Bie Z, et al. Melatonin: Current Status and Future Perspectives in Plant Science. Front Plant Sci. 2016;6:1230.

5. Kaur H, Mukherjee S, Baluska F, et al. Regulatory roles of serotonin and melatonin in abiotic stress tolerance in plants. Plant Signaling \& Behavior. 2015;10(11):e1049788. 